\title{
Sr. Realista Estructural, Tenemos un Problema: LA CARGA ONTOLÓGICA DE LAS MATEMÁTICAS
}

\author{
Carlos M. Madrid CASAdo \\ Universidad Complutense de Madrid
}

\begin{abstract}
The aim of this note is to undermine structural realism by testing the soundness of its main claim. If scientific theories represent the structure of the world, structural realism needs a general account of representation. Representation is the crux of structural realism, because structure/ontology distinction collapses. Mathematical structures are ontologyladen.
\end{abstract}

Keywords: Structural realism, scientific representation, structure/ontology distinction, quantum theory, matrix and wave mechanics.

Cuando hay algo que no anda bien en vuestra epistemología, es que hay algo que no anda bien en vuestra ontología.

A. N. Whitehead

\section{Problemas abiertos del realismo estructural}

Hace ya dos décadas, John Worrall (1989) dio carta de naturaleza a ese realismo científico mínimo que denominó realismo estructural y que se encuentra a medio camino entre el realismo clásico y el instrumentalismo. El realista estructural defiende, por una parte, que el realismo es la única filosofía que no hace del éxito de la ciencia un milagro. Si las teorías científicas no fueran aproximadamente verdaderas en algún sentido, sería un milagro que realizaran predicciones tan acertadas. Pero, el realista estructural reconoce, por otra parte, que múltiples teorías científicas, que en su momento fueron consideradas como verdaderas a la luz de su éxito, han sido posteriormente refutadas y abandonadas. La cuestión es cómo acomodar, a un mismo tiempo, la "inferencia a la mejor explicación" y la "inducción pesimista".

Worrall encontró la solución en Poincaré: a través del cambio científico, hay continuidad; pero esa continuidad no es de la ontología sino de la estructura. El mobiliario que atribuimos al mundo cambia notablemente, pero conserva su disposición. Pese a que la ontología individual sufre cambios radicales, las teorías nuevas retienen la estructura matemática de las teorías antiguas, porque las estructuras matemáticas de las teorías científicas representan la estructura del mundo. Ésta es la clave del realismo estructural: "las ecuaciones expresan relaciones y si las ecuaciones siguen siendo verdaderas es porque las relaciones siguen siendo reales" (Worrall 1989, p. 118).

Principia 14(2): 201-209 (2010).

Published by NEL — Epistemology and Logic Research Group, Federal University of Santa Catarina (UFSC), Brazil. 
Ahora bien, mientras que el realismo estructural defendido por Worrall (1989 y 1994) es de corte epistemológico, por cuanto su punto de vista es que sólo podemos conocer la estructura del mundo (permaneciendo incognoscible su ontología), el realismo estructural de nuestros días - propuesto por Steven French y James Ladyman (2003) - toma partido por una posición ontológica muy determinada. Este nuevo realismo estructural abraza — por decirlo con Psillos (2006, p. 561) - un "estructuralismo óntico", que consiste en creer que "todo lo que hay es estructura" y que "nuestras teorías científicas son capaces de capturar las estructuras existentes en el mundo" (Da Costa \& French 2003, p. 189). Las estructuras serían, por así decirlo, sustancias ontológicas primitivas.

En cualquier caso, tanto la variedad epistémica como la variedad óntica del realismo estructural concuerdan en tres tesis básicas:

i) Las estructuras matemáticas sobreviven al cambio de teorías científicas.

ii) Las teorías científicas nos desvelan, por medio de su estructura matemática, la estructura de la realidad.

iii) Una estructura matemática es compatible con diferentes ontologías de individuos.

Precisamente, cada una de estas tesis puede ponerse en correspondencia con uno de los problemas que tiene abiertos el realismo estructural:

(Problema 1.) El realismo estructural da por hecho que existe una continuidad matemática en la sucesión de teorías científicas (las ecuaciones se preservan o perviven, supuestamente, como casos límite); pero la historia de la ciencia aporta episodios en que la estructura matemática, como la ontología, no parece que haya sobrevivido.

(Problema 2.) El realismo estructural mantiene que la estructura matemática de la teoría es capaz de representar, en sentido realista (no meramente empirista), la estructura del mundo. Pero si las teorías científicas representan - por medio de su estructura matemática- la estructura de la realidad, los realistas estructurales tienen qué explicar que noción de representación emplean para poder garantizar la inferencia realista ("las teorías capturan la estructura del mundo").

(Problema 3.) El realismo estructural acepta, por principio, que podemos distinguir, separar y disociar una estructura y una ontología en nuestras teorías científicas.

Curiosamente, la primera cuestión ha sido la que mayor atención ha recibido por parte de los críticos del realismo estructural, quedando la segunda y la tercera en un segundo plano. El objetivo de esta nota es argumentar que la cuestión de la 
representación científica es, de hecho, la principal fisura del realismo estructural. Y su análisis lleva a cuestionar el dogma de la distinción estructura/ontología.

\section{Representación científica y estructuralismo realista}

El estructuralismo (sea realista o empirista) sostiene que las teorías y los modelos nos informan de la estructura del mundo. El estructuralismo realista añade, además, que nos informan de la estructura inobservable de la realidad. En consecuencia, de acuerdo con French y Saatsi (2006, p. 556), "el realismo estructural es la concepción de que nuestras mejores teorías representan el mundo de modo aproximadamente correcto" (cursivas mías). Si las teorías científicas no representasen la estructura de la realidad, el éxito de la ciencia sería un milagro. Pero, ¿qué significa exactamente "representar la estructura del mundo o de la realidad"?

La noción de representación plantea un problema al realismo estructural. De hecho, Van Fraassen (2006a, pp. 536-9) acepta que el empirismo constructivo comparte este problema con el realismo estructural, puesto que también consiste en un estructuralismo, sólo que esta vez empirista: un modelo es empíricamente adecuado si y sólo si representa la estructura de las apariencias. Van Fraassen (2002, p. 252) defiende que, si adoptamos su postura empirista, el problema puede solucionarse fácilmente: la estructura de la teoría no está representando la estructura del mundo, ni siquiera la estructura de las apariencias (algo que tampoco es abstracto), sino simplemente la estructura del modelo abstracto de los datos empíricos, que el científico elabora registrando sus observaciones. Ahora bien, esto no resuelve el problema, sino que únicamente lo desplaza: ¿cómo representan los modelos de los datos la estructura de los fenómenos? El estructuralismo, sea de signo empirista o realista, necesita — como señalan Brading y Landry (2006, p. 578) — precisar bien qué entiende por representación.

La tentación pitagórica consiste en cortar por lo sano y replicar que la estructura matemática de la teoría simplemente está copiando o reflejando la propia estructura matemática del mundo, como si la realidad estuviera escrita en caracteres matemáticos. Pero el realista estructural puede hacerlo mucho mejor. Cuando afirma que la teoría representa el mundo quiere decir que la teoría y el mundo comparten, en cierto grado, la misma estructura. De otra manera: la estructura (matemática) de la teoría y la estructura (matematizable) del mundo son (parcialmente) isomorfas (French 2003; Bueno, French y Ladyman 2002; Da Costa y French 2003). Ahora bien, ¿no nos conducirá este postulado de isomorfismo estructural entre la teoría y el mundo a un callejón sin salida?

Para contestar, vamos a servirnos como piedra de toque del estudio de la equivalencia matemática entre Mecánicas Cuánticas (Madrid Casado: 2008). Pero antes,

Principia 14(2): 201-209 (2010). 
necesitamos realizar algunas precisiones terminológicas al hilo de lo dicho en los párrafos precedentes. Dentro de los límites de esta nota distinguimos tres clases de estructuras. Por un lado, en el ámbito teórico, las estructuras —abstractas - de la teoría y de los datos (los modelos). (Por simplificar únicamente vamos a hablar de estructuras teóricas —-matemáticas-.) Por otro, en el mundo, las estructuras empíricas (fenoménicas, aparentes) y las estructuras ónticas (inobservables) de la realidad. Así pues, aparecen tres niveles: estructuras teóricas (matemáticas), empíricas y ónticas. ${ }^{1}$ De este modo, para el empirista estructural, la meta de la ciencia es salvar la estructura empírica u observable de la realidad. En cambio, el realista estructural sustenta que las estructuras teóricas, aunque no capturen la ontología del mundo, aspiran a representar su estructura óntica; por cuanto esta última, que yace detrás de los fenómenos y las apariencias, es causa de la estructura empírica de la realidad.

Tras años de trabajo, hacia 1932, Von Neumann construyó la primera prueba de equivalencia matemática rigurosa y completa entre la Mecánica Matricial (MM) de Heisenberg-Born-Jordan, 1925, y la Mecánica Ondulatoria (MO) de Schrödinger, 1926. Von Neumann reconstruyó MM y MO como sendos cálculos de operadores sobre espacios de Hilbert (respectivamente, sobre el espacio de las sucesiones de cuadrado sumable - las columnas de las matrices - y sobre el espacio de las funciones de cuadrado integrable - las funciones de onda-), para a continuación demostrar que la Mecánica de Heisenberg (centrada en matrices y sumas) y la Mecánica de Schrödinger (centrada en funciones e integrales) son matemáticamente equivalentes al no ser más que dos cálculos de operadores isomorfos sobre dos espacios de Hilbert isomorfos (isométricos).

Las estructuras teóricas —-matemáticas- de MM y MO eran equivalentes (isomorfas), y esta relación de equivalencia por isomorfismo garantizaba la deducción de idénticas predicciones empíricas, algo que los físicos cuánticos ya habían constatado en la práctica (MM y MO arrojaban, por ejemplo, los mismos valores energéticos para el átomo de hidrógeno). Las estructuras empíricas de MM y MO eran, también, equivalentes.

Sin embargo, las estructuras ónticas que MM y MO prescribían a la realidad no eran, atención, equivalentes (isomorfas). En efecto, MM proyecta sobre el mundo una estructura discreta, dado que su realización del espacio de Hilbert —el espacio de las sucesiones de cuadrado sumable - es discreta, lo que llevaba a Born y Jordan a referirse a MM como una "verdadera teoría del discontinuo" y acentuaba una visión corpuscular del mundo atómico. En cambio, MO proyecta sobre la realidad una estructura continua, dado que su realización del espacio de Hilbert —el espacio de las funciones de cuadrado integrable- es continua, lo que provocaba que Schrödinger hablara de MO como de una "nueva física del continuo" y casaba con una concepción ondulatoria del microcosmos.

Ahora bien, desde el punto de vista del realista estructural, la estructura mate-

Principia 14(2): 201-209 (2010). 
mática de la teoría captura (isomórficamente) la estructura óntica de la realidad. Por tanto, si dos teorías son matemáticamente equivalentes, esto es, si sus estructuras matemáticas son isomorfas, el realista estructura está obligado a esperar que sus estructuras ónticas —al igual que las empíricas - también sean isomorfas. Con otros términos: ambas teorías tienen que ofrecer una misma estructura subyacente de la realidad. Pero, como va dicho, las estructuras matemáticas y empíricas de MM y MO son isomorfas y, sin embargo, las estructuras ónticas que MM y MO proyectan sobre la realidad no lo son ni pueden serlo, porque una es discreta y la otra es continua. Por encima de que MM y MO nos dibujen un mundo poblado de corpúsculos u ondas (con distinta ontología), ambas teorías ya nos están (re-)presentando dos mundos estructuralmente incompatibles (discreto versus continuo). ${ }^{2}$

En suma, la noción de representación supone un problema para el realista estructural, si se concibe en términos de isomorfismo y afectando a lo inobservable (es decir, en sentido realista). De hecho, los propios Bueno, French y Ladyman (2002, p. 503) reconocen que este "argumento de la cardinalidad" es una amenaza seria tanto para isomorfismo como isomorfismo parcial. Y, ¿qué sucede si interpretamos la noción de representación de un modo más débil? Bueno, French y Ladyman (2002) conciben la relación de representación como homomorfismo (parcial). La objeción es que esta noción, aunque desactiva el "argumento de la cardinalidad" (los homomorfismos no tienen por qué ser biyecciones), es demasiado débil para legitimar la inferencia realista. Si la relación entre la teoría y el mundo se caracteriza empleando homomorfismos, se garantiza la deducción de arriba abajo (teoría $\rightarrow$ mundo) pero no de abajo arriba (mundo $\rightarrow$ teoría), puesto que los homomorfismos no son en general invertibles. En consecuencia, la inferencia realista estructural ("la teoría captura la estructura del mundo") es ilegítima. Sin una relación representacional fuerte (de isomorfismo), los realistas estructurales tienen muy difícil establecer inferencias sobre la estructura óntica de la realidad.

\section{La carga ontológica de las estructuras matemáticas}

Las consideraciones precedentes acerca de la representación realista de estructuras nos conducen al tercer punto, a cuestionar la distinción estructura/ontología (forma/contenido, formalismo/interpretación) del realismo estructural. Según vamos a argumentar, no existe un corte limpio entre la estructura y la ontología de nuestras teorías, porque las estructuras matemáticas están cargadas de ontología ...

Fue Worrall (1989, p. 117) quien acuñó esta distinción, analizando la evolución de las ecuaciones en óptica: "Fresnel identificó de modo completamente erróneo la naturaleza [ontología] de la luz, pero no es ningún milagro que su teoría disfrutase del éxito empírico predictivo que tuvo; no es ningún milagro, porque la teoría de

Principia 14(2): 201-209 (2010). 
Fresnel, como lo vio la ciencia posterior, atribuyó a la luz la estructura correcta". La estructura vendría dada por el formalismo matemático de la teoría; y la ontología, por la interpretación física. Pero, ¿es siempre posible demarcar con precisión la estructura de la ontología?

A mi entender, no existe corte entre ambas y, en consecuencia, no se puede ser realista con respecto a las estructuras y, simultáneamente, antirrealista con respecto a las entidades que contienen. El "semirrealismo", como lo denomina Chakravartty (1998), es altamente inestable; porque uno no puede creer que ciertas relaciones son reales a menos que también acepte que ciertos objetos están relacionados así. La distinción entre la verdad de las relaciones y la verdad acerca de los relata no marcha. Por decirlo en unos términos escolásticos que el propio realista estructural resucita: la forma es inseparable de la materia. French y Ladyman (2003, p. 37) responden divorciando el realismo estructural de cualquier compromiso con ontologías de individuos: "una formulación del realismo adecuada a la física precisa estar construida sobre la base de una ontología alternativa que reemplace la noción de objeto por la de estructura". El mundo no consistiría en objetos, sino sólo en estructuras. El realismo estructural óntico radicaliza — como observa Van Fraassen (2006b) — el estructuralismo del realismo estructural epistémico hasta el punto de "reificar" las propias estructuras. Pero, ¿es aceptable la desaparición de las entidades, un mundo sin objetos? La indistinguibilidad de las partículas cuánticas mostraría, para sus partidarios, que referirse a ellas es sólo un modo de hablar. Pero los nodos de las estructuras cuánticas pueden no ser meros fantasmas, sino objetos de una categoría diferente a los macroscópicos. ¿O acaso estos últimos (que podemos señalar y agarrar) tampoco existen, son apariencias? Como concluye Van Fraassen (2007a, p. 55): "¿Tiene sentido concebir una estructura que no es estructura de algo? Una estructura de nada es nada".

Pero hay más: la ocurrencia del realista estructural de que una misma estructura matemática es compatible con ontologías muy diferentes dista de ser cierta, ya que toda estructura lleva aparejada una carga ontológica. Las estructuras matemáticas están cargadas de ontología. La estructura matemática no puede separarse con nitidez de la ontología física, porque no existe algo así como una neutralidad ontológica de las matemáticas. Ningún lenguaje —ni siquiera el matemático- es neutro en la descripción del mundo.

En efecto, la matemática algebraica de MM apoyaba una ontología discreta, corpuscular. En cambio, la matemática analítica de MO sugería una ontología continua. Si había una ecuación de ondas, tenía que haber ondas o algo similar. Las estructuras matemáticas de MM y MO son equivalentes pero, al ser de muy distinta raigambre, contienen subestructuras ónticas no isomorfas (discreta $v s$. continua) que apuntan a ontologías incompatibles (corpuscular vs. ondulatoria). Confiado en la realidad de la estructura abstracta del espacio de Hilbert, el realista estructural no tiene más

Principia 14(2): 201-209 (2010). 
opción que ser realista tanto con respecto a la realización discreta como con respecto a la continua; a la manera que el matemático formalista, que trabaja clausurado en su sistema axiomático, no tiene más que aceptar que los números reales poseen tanto modelos estándar — no numerables, continuos- como modelos no estándar -numerables, discretos- (la física reproduce la principal aporía de la matemática: ¿es continuo o discreto el fondo de la naturaleza?). Sin embargo, este paso entraña numerosas dificultades, porque no hay una única estructura óntica ni una única ontología del mundo cuántico asociada con ambos tipos de estructura matemática (dualidad onda-corpúsculo). No basta con aferrarse al formalismo y afirmar que las estructuras matemáticas que éste satisface son las únicas en que cree el realista estructural, porque cada estructura matemática puede conllevar una subestructura óntica distinta.

El formalismo condiciona y, a veces, determina la interpretación ${ }^{3}$, porque el aparato matemático empleado arrastra un peso ontológico. No quiero implicar que la estructura matemática fije de una vez por todas la ontología, pero sí que restringe bastante la clase de ontologías compatibles con el formalismo (con más precisión: compatibles con la subestructura óntica coordinable con el formalismo). La historia de la Mecánica Cuántica nos ofrece más ejemplos de este condicionamiento. En la formulación de la Mecánica Cuántica obra de Feynman, la utilización de la herramienta matemática conocida como integral de camino soporta la interpretación como suma de historias. En la Mecánica Bohmiana, la propiedad de existencia y unicidad global de solución de la ecuación de puntos-guía, que implica que las trayectorias no pueden cortarse ni fusionarse, posibilita la interpretación realista y determinista. O, por no seguir, la interpretación de Born o la de De Broglie, que borran la contradicción entre partículas y ondas, se abrieron paso gracias a la modificación del formalismo (mediante la introducción de medidas de probabilidad y la teoría matemática de la doble solución, respectivamente). Cambian la interpretación y la ontología, porque cambian las estructuras matemáticas. La tarea del físico teórico no se limita a buscar una teoría matemática adecuada a la experiencia, porque el repertorio de teorías matemáticas que tiene a su disposición orienta decisivamente el alcance ontológico. La física matemática es, según la conocida expresión de Meyerson, creadora de ontología.

En resumen, la distinción estructura/ontología colapsa, porque -como afirma Psillos (1995, p. 31, y 1999, p. 157) - ambas forman un continuo. La carga ontológica de las matemáticas no es despreciable y, por consiguiente, la posibilidad de diseccionar la estructura matemática de la ontología de las teorías físicas, como si fueran dos mundos completamente independientes, parece un espejismo. 


\section{Conclusiones}

Aparte de dejar constancia de las fisuras del realismo estructural con respecto al tópico de la representación científica, he tratado de argumentar que las estructuras no pueden separarse de las ontologías, pergeñando una tesis que atenta directamente contra la viabilidad del realismo estructural: la carga ontológica de la matemática.

Una conexión a explorar es la de esta tesis con el argumento de indispensabilidad de Quine-Putnam: en tanto en cuanto las estructuras matemáticas se muestran indispensables para nuestras teorías físicas, pues contribuyen tan genuinamente como lo hacen las partículas hipotéticas, comparten su estatus ontológico. Desde esta perspectiva holista, cuando contrastamos una teoría con la experiencia, no sólo estamos poniendo a prueba las hipótesis físicas, sino también las estructuras matemáticas utilizadas. No en vano, como notara Poincaré, el indeterminismo cuántico rompió el matrimonio entre la física y las ecuaciones diferenciales, al introducir discontinuidades que requerían otra matemática. La matemática puede verse afectada, ontológicamente hablando, por la suerte que corra la física en cuya formulación aparece. Quizá, sencillamente, por la razón de que también aporta su grano de ontología.

\section{Referencias}

Brading, K. \& Landry, E. 2006. Scientific Structuralism: Presentation and Representation. Philosophy of Science 73: 571-81.

Bueno, O., French, S. \& Ladyman, J. 2002. On representing the relationship between the mathematical and the empirical. Philosophy of Science 69: 497-518.

Chakravartty, A. 1999. Semirealism. Stud. His. Phil. Sci. 29: 391-408.

Da Costa, N. \& French, S. 2003. Science and Partial Truth. Oxford: Oxford University Press.

French, S. 2003. A Model-Theoretic Account of Scientific Representation (Or, I Don't Know Much About Art. . But I Know It Involves Isomorphism). Philosophy of Science 70: 147283.

French, S. \& Ladyman, J. 2003. Remodelling Structural Realism: Quantum Physics and the Metaphysics of Structure. Synthese 136: 31-56.

French, S. \& Saatsi, J. 2006. Realism about Structure: The Semantic View and Nonlinguistic Representations. Philosophy of Science 73: 548-559.

Hanson, N. R. 1961. Are wave mechanics and matrix mechanics equivalent theories? En H. Feigl \& G. Maxwell (eds.) Current issues in the philosophy of science. Nueva York: Holt Rinehart and Winston.

Madrid Casado, C. M. 2008. A brief history of the mathematical equivalence between the two quantum mechanics. Lat. Amer. J. Phys. Ed. 2(2): 104-8.

Psillos, S. 1995. Is structural realism the best of both worlds?. Dialectica 49: 15-46.

- 1999. Scientific Realism: How Science Tracks Truth. Londres: Routledge.

. 2006. The Structure, the Whole Structure, and Nothing but the Structure. Philosophy of Science 73: 560-70.

Principia 14(2): 201-209 (2010). 
Van Fraassen, B. 2002. The Empirical Stance. New Haven: Yale University Press.

- 2006a. Representation: The Problem for Structuralism. Philosophy of Science 73: $536-47$.

- 2006b. Structure: Its Shadow and Substance. Brit. J. Phil. Sci. 57: 275-307.

- 2007a. Structuralism(s) about Science: Some Common Problems. Proc. Arist. Soc. 81: 45-61.

- 2007b. Representation and Perspective in Science. Principia 11(2): 97-116.

Votsis, S. 2007. Uninterpreted Equations and the Structure-Nature Distinction. Philosophical Inquiry 29: 57-71.

Worrall, J. 1989. Structural Realism: The Best of Both Worlds? Dialectica 43: 99-124. . 1994. How to Remain (Reasonably) Optimistic: Scientific Realism and the "Luminiferous Ether". En D. Hull, M. Forbes \& R. M. Burian (eds.) PSA 1994 Vol. 1. East Lansing: Philosophy of Science Association, East Lansing.

$$
\begin{array}{r}
\text { CARlos M. MADRID CASADO } \\
\text { Departamento de Matemáticas } \\
\text { Instituto Lázaro Cárdenas } \\
\text { C/ Isla de Sálvora } 153 \text { \#28400\# Collado Villalba } \\
\text { Madrid }
\end{array}
$$

Departamento de Estadística e Investigación Operativa II

Universidad Complutense de Madrid

Campus de Somosaguas \#28223\# Madrid

EsPaña

cmadrid@ccee.ucm.es

Resumo. O objetivo desta nota é solapar o realismo estrutural testando a correção de sua alegação principal. Se teorias científicas representam a estrutura do mundo, o realismo estrutural necessita de uma explicação geral da representação. Representação é o ponto crucial do realismo estrutural, pois a distinção estrutura/ontologia colapsa. Estruturas matemáticas são carregadas de ontologia.

Palavras-chave: Realismo estrutural, representação científica, distinção estrutura/ontologia, teoria quântica, mecânica matricial e ondulatória.

\section{Notas}

${ }^{1}$ Si comparamos nuestra clasificación con la de Van Fraassen (2007b, pp. 104-5), observamos que el dominio "realidad postulada teoréticamente" contiene las estructuras ónticas, mientras que son los dominios de "fenómenos observables" y de "apariencias" los que incluyen las estructuras empíricas.

${ }^{2}$ Hace ya bastante tiempo que Norwood R. Hanson (1961), en "Are wave mechanics and matrix mechanics equivalent theories?", llamó la atención sobre las maneras en que la equivalencia formalmente demostrada puede, a pesar de todo, permitir usos, fines y formas muy diferentes de entender las distintas "formulaciones".

${ }^{3}$ En sentido ontológico, pues con respecto a la estructural y a la puramente observacional el realista estructural no es agnóstico (Votsis 2007).

Principia 14(2): 201-209 (2010). 